\title{
Dermolipoma e prolapso de gordura orbital - duas entidades distintas
}

\author{
Dermolipoma and orbital fat prolapse-twodistinct entities
}

\author{
Lucieni Cristina Barbarini Ferraz ${ }^{1}$ \\ Silvana Artioli Schellini ${ }^{2}$ \\ Sheila Cristina Lordelo Wludarski ${ }^{3}$ \\ Mariângela Esther Alencar Marques ${ }^{4}$ \\ Maria Rosa Bet Moraes-Silva ${ }^{5}$
}

\section{RESUMO}

Objetivo: Relatar 20 pacientes portadores de dermolipoma e 10 pacientes com prolapso de gordura orbitária, ressaltando aspectos que podem auxiliar para o diferencial clínico destas duas entidades. Métodos: Foi realizado estudo retrospectivo de 12 anos, avaliando-se portadores de dermolipoma e de prolapso de gordura orbitária, atendidos na Faculdade de Medicina de Botucatu-SP. Resultados: No período foram detectados 20 (1,6 pacientes/ano) portadores de dermolipoma e $10(0,8$ pacientes/ano), de prolapso de gordura orbitária. Quanto ao sexo, o dermolipoma acometeu mais mulheres e o prolapso de gordura orbital ocorreu mais em homens. Nos portadores de dermolipoma, a lesão foi encontrada no canto externo em todos os pacientes, sendo bilateral em apenas um caso; nos com prolapso de gordura orbital, a lesão localizava-se no canto externo em 9 dos 10 portadores. Sete pacientes com dermolipoma possuíam associação com outras doenças oculares e em dez pacientes a lesão estava presente desde o nascimento. Conclusão: $\mathrm{O}$ dermolipoma é semelhante ao prolapso de gordura orbitária quanto à localização e aparência clínica. Porém, o dermolipoma está presente desde o nascimento, ocorre mais no sexo feminino, podendo estar associado a outras doenças oculares. O prolapso de gordura orbitária é alteração que ocorre em indivíduos idosos, geralmente do sexo masculino.

Descritores: Lipoma; Tecido adiposo; Coristoma/diagnóstico; Prolapso; Doenças da órbita/diagnóstico; Diagnóstico diferencial; Estudos retrospectivos

${ }^{1}$ Residente de Oftalmologia- Depto. de Oftalmologia, Otorrinolaringologia e Cirurgia de Cabeça e PescoçoFaculdade de Medicina de Botucatu - Universidade Estadual Paulista.

2 Professor Adjunto- Depto. de Oftalmologia, Otorrinolaringologia e Cirurgia de Cabeça e Pescoço- Faculdade de Medicina de Botucatu - Universidade Estadual Paulista.

${ }^{3}$ Residente de Patologia - Depto. de Patologia - Faculdade de Medicina de Botucatu - Universidade Estadual Paulista.

${ }^{4}$ Professor Assistente - Doutor - Depto. de Patologia Faculdade de Medicina de Botucatu - Universidade Estadual Paulista.

${ }^{5}$ Professor Assistente - Doutor- Depto. de Oftalmologia, Otorrinolaringologia e Cirurgia de Cabeça e Pescoço- Faculdade de Medicina de Botucatu - Universidade Estadual Paulista.

Endereço para correspondência: Depto. Oftalmologia, Otorrinolaringologia e Cirurgia de Cabeça e Pescoço Faculdade de Medicina de Botucatu - Universidade Estadual Paulista - UNESP - Botucatu (SP) CEP 18618-000. E-mail: sartioli@fmb.unesp.br

Nota Editorial: Pela análise deste trabalho e por sua anuência sobre a divulgação desta nota, agradecemos ao Dr. Paulo Góis Manso.

\section{INTRODUÇ̃̃O}

O dermolipoma é um tumor do tipo disgenético, raro, benigno, em geral presente ao nascimento. Possui aspecto clínico característico, o que permite o seu reconhecimento sem dificuldade.

Existe, porém, uma lesão muito parecida com o dermolipoma, que surge na idade adulta e que pode ser confundida com aquele, que é o prolapso de gordura orbitária.

Clinicamente ambas as entidades são vistas como lesão de coloração amarelada, homogênea, coberta por conjuntiva de superfície lisa, contendo poucos vasos e sem sinais inflamatórios. Devido à semelhança clínica, há dificuldade para diferenciar estas duas entidades, o que já foi realçado por dois estudos anteriores ${ }^{(1-2)}$.

O presente estudo teve por objetivo ressaltar os aspectos que podem auxiliar o diferencial clínico destas duas afecções. 


\section{MÉTODOS}

Foi realizado estudo retrospectivo dos pacientes com diagnóstico clínico de dermolipoma e de prolapso de gordura orbitária, atendidos na Faculdade Medicina de Botucatu - Universidade Estadual Paulista - UNESP, no período de 1988 até 2000, avaliando-se: idade, cor da pele, sexo, queixa, tempo de evolução, tamanho e localização da lesão, diagnóstico clínico, diagnóstico histopatológico (nos casos em que foi feita exérese da lesão), associação com outras patologias e complicações.

\section{RESULTADOS}

Os dados referentes aos pacientes se encontram nos quadros 1 e 2 .

Foram encontrados 20 pacientes com diagnóstico clínico de dermolipoma (Figura 1) e 10, com prolapso de gordura orbitária (Figura 2).

A idade dos pacientes variou de 6 meses a 40 anos (média de 12 anos) nos portadores de dermolipoma, e 54 a 76 anos nos com prolapso da gordura orbitária (média de 64,8 anos).

Entre os pacientes com dermolipoma houve acometimento de 12 mulheres e 8 homens, sendo 19 brancos e 1 negro. O prolapso de gordura orbitária foi observado em 7 homens e 3 mulheres, sendo 9 brancos e 1 negro.

Os pacientes procuraram tratamento 6 meses e até 26 anos após a detecção do dermolipoma. Dez pacientes relataram a presença da lesão desde o nascimento. Pacientes com prolapso da gordura orbitária procuraram o serviço entre 2 meses a 17 anos após o aparecimento da lesão.

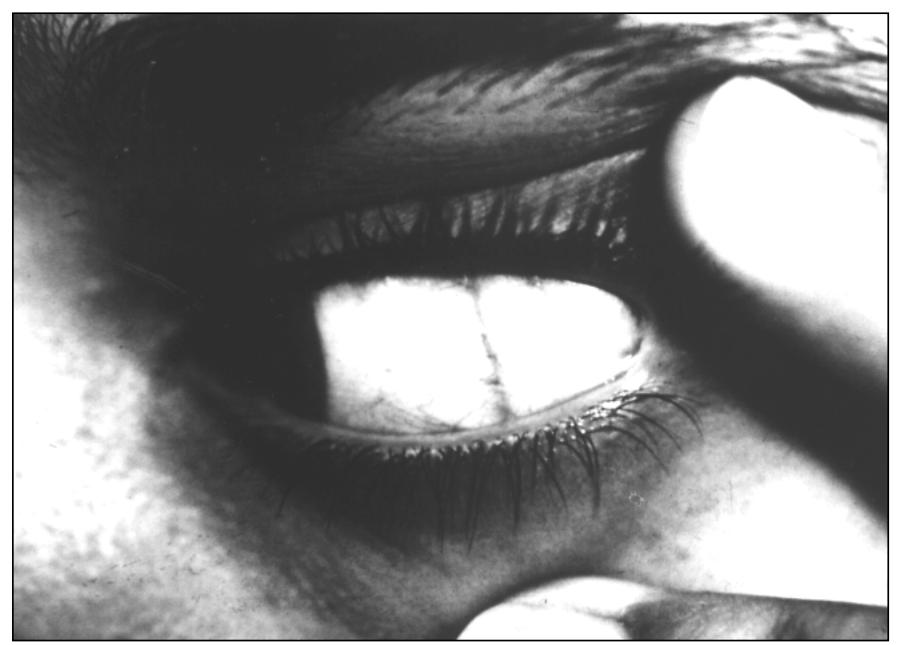

Figura 1 - Paciente portador de dermolipoma

O dermolipoma se localizava no canto externo em todos os casos; 7 no lado direito e 11 no esquerdo (um deles não havia descrição da localização). O prolapso de gordura orbitária foi encontrado em 9 pacientes no canto externo e um deles apresentou a lesão no canto interno.

O dermolipoma esteve associado a outras patologias oculares em 7 pacientes: olho seco, glaucoma congênito, simbléfaro, alterações congênitas (hipertelorismo, nariz em sela, fronte olímpica, língua protusa), estrabismo, catarata congênita e conjuntivite alérgica. O prolapso de gordura orbitária não teve associação com outras patologias.

A exérese da lesão foi realizada em 6 pacientes com dermolipoma e em 8 com prolapso de gordura orbitária. Em todas as

\begin{tabular}{|c|c|c|c|c|c|c|c|c|}
\hline \multicolumn{9}{|c|}{ Quadro 1. Apresentação dos portadores de dermolipoma } \\
\hline $\mathbf{N}$ & Idade & Cor & Sexo & Queixa & Tempo & Local & Associação & Cirurgia \\
\hline 1 & $20 a$ & B & $\mathrm{F}$ & Ardor & - & $\mathrm{CeOe}$ & Olho seco & $\mathrm{N}$ \\
\hline 2 & $40 a$ & B & $\mathrm{F}$ & A lesão & - & $\mathrm{CeOd}$ & - & $\mathrm{S}$ \\
\hline 3 & $26 a$ & $\mathrm{~B}$ & $\mathrm{~F}$ & A lesão & 3 & $\mathrm{CeOe}$ & Glaucoma congênito & $S$ \\
\hline 4 & $8 m$ & B & $\mathrm{F}$ & A lesão & $8 m$ & $\mathrm{CeOe}$ & - & $\mathrm{N}$ \\
\hline 5 & $32 a$ & B & M & A lesão & $3 m$ & & - & \\
\hline 6 & $17 a$ & B & $\mathrm{F}$ & A lesão & $6 m$ & $\mathrm{CeOd}$ & Simbléfaro & \\
\hline 7 & $1 a$ & B & M & A lesão & $1 a$ & $\mathrm{CeOd}$ & Hipertelorismo & $\mathrm{N}$ \\
\hline 8 & $6 m$ & B & M & A lesão & $6 m$ & $\mathrm{CeOd}$ & - & $\mathrm{N}$ \\
\hline 9 & $10 a$ & $\mathrm{~N}$ & $\mathrm{~F}$ & A lesão & $10 a$ & $\mathrm{CeOe}$ & - & $\mathrm{N}$ \\
\hline 10 & $16 a$ & B & $\mathrm{F}$ & Edema & $16 a$ & $\mathrm{CeOe}$ & - & $S$ \\
\hline 11 & $10 a$ & B & $\mathrm{F}$ & A lesão & $10 a$ & $\mathrm{CeOe}$ & - & $\mathrm{N}$ \\
\hline 12 & $6 m$ & B & $\mathrm{F}$ & A lesão & $6 m$ & $\mathrm{CeOd}$ & - & $\mathrm{N}$ \\
\hline 13 & $9 m$ & B & M & A lesão & $9 m$ & $\mathrm{CeOe}$ & - & $\mathrm{N}$ \\
\hline 14 & $9 m$ & B & M & A lesão & $3 m$ & $\mathrm{CeOe}$ & - & $\mathrm{N}$ \\
\hline 15 & $4 a$ & B & M & A lesão & $4 a$ & $\mathrm{CeOd}$ & - & $\mathrm{N}$ \\
\hline 16 & 26 & B & $\mathrm{F}$ & Cisto OD & $26 a$ & $\mathrm{CeOd}$ & Simbléfaro & $\mathrm{S}$ \\
\hline 17 & $1 a$ & B & M & Olho torto & $1 a$ & $\mathrm{CeOe}$ & Estrabismo & $\mathrm{N}$ \\
\hline 18 & $14 a$ & B & $\mathrm{F}$ & Ardor & $2 a$ & CeAo & Cat congênita & $\mathrm{S}$ \\
\hline 19 & $8 a$ & B & M & Prurido & $4 a$ & $\mathrm{CeOe}$ & Conj Alérgica & $\mathrm{N}$ \\
\hline 20 & $12 \mathrm{a}$ & B & $\mathrm{F}$ & Cisto, desvio ocular & $6 m$ & $\mathrm{CeOe}$ & Hipotireoidismo & $S$ \\
\hline
\end{tabular}




\begin{tabular}{|c|c|c|c|c|c|c|c|c|}
\hline \multicolumn{9}{|c|}{ Quadro 2. Apresentação dos portadores de prolapso de gordura orbitária } \\
\hline $\mathbf{n}$ & idade & cor & sexo & queixa & tempo & Local & Associação & Cirurgia \\
\hline 1 & $76 a$ & B & M & cisto & $1 \mathrm{a}$ & CeAo & - & $\mathrm{S}$ \\
\hline 2 & $58 a$ & B & M & A lesão & $2 m$ & $\mathrm{CeOe}$ & Cisto pilonidal & $S$ \\
\hline 3 & $68 a$ & B & $\mathrm{F}$ & A lesão & $2 a$ & $\mathrm{CeOe}$ & - & $S$ \\
\hline 4 & $59 a$ & B & $\mathrm{F}$ & raspar & - & $\mathrm{CeOd}$ & Triquíase & $S$ \\
\hline 5 & $54 a$ & $\mathrm{~N}$ & M & A lesão & $4 m$ & $\mathrm{CeOe}$ & - & $S$ \\
\hline 6 & $60 a$ & $\mathrm{Nb}$ & $M$ & A lesão & $17 a$ & $\mathrm{CiOe}$ & - & $S$ \\
\hline 7 & $76 a$ & B & $M$ & A lesão & $1 a$ & CeAo & - & $S$ \\
\hline 8 & $70 a$ & B & M & A lesão & $6 m$ & $\mathrm{CeOe}$ & - & $S$ \\
\hline 9 & $70 a$ & B & $\mathrm{F}$ & $<\mathrm{AV}$ & - & $\mathrm{CeOd}$ & - & $\mathrm{N}$ \\
\hline 10 & $57 a$ & B & $M$ & A lesão & $2 m$ & $\mathrm{CeOe}$ & - & $\mathrm{N}$ \\
\hline
\end{tabular}

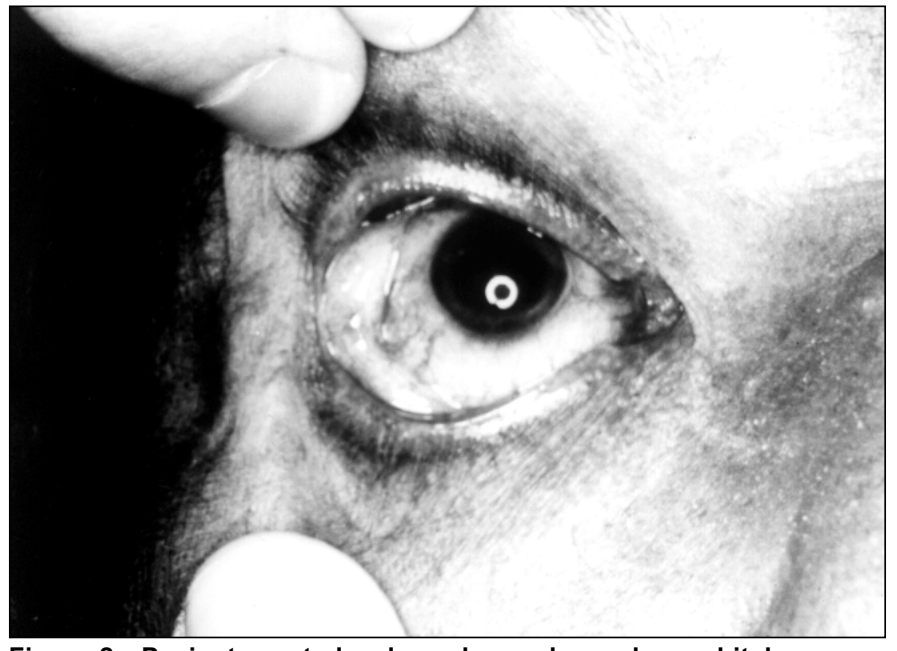

Figura 2 - Paciente portador de prolapso de gordura orbital

ressecções, a exérese foi incompleta, removendo-se apenas a porção anterior e aparente da lesão.

Apesar da aparência clínica semelhante, as duas entidades diferiram ao exame histopatológico. O dermolipoma foi visto como uma lesão coberta por epitélio escamoso estratificado, com estroma subepitelial contendo feixes de tecido colágeno denso, onde foi observado em um dos pacientes a presença de estruturas pilosebáceas (Figura 3A). No estroma profundo, presença de tecido adiposo disposto em lóbulos separados entre si por traves de tecido colágeno denso (Figura 3B). Já, o prolapso de gordura orbital foi visto como composto por adipócitos maduros (Figura 4 ).

\section{DISCUSSÃO}

Dermolipomas são coristomas, geralmente localizados no canto externo e estão presentes desde o nascimento ${ }^{(3)}$. Geralmente são móveis, macios e indolores. $\mathrm{O}$ aspecto clínico é amarelo e fusiforme, localizado na conjuntiva temporal, próximo ao canto externo.

Os lipomas e os lipossarcomas podem ser clinicamente semelhantes. Porém, estes últimos cursam com outras alterações, inclusive sistêmicas, que permitem diferenciá-los dos primeiros.

Os tumores de lipócitos verdadeiros são extremamente raros na órbita, a despeito da presença abundante de gordura nesta região. Um estudo verificou que dentre 222 tumores orbitários, apenas 19 eram lipomas ${ }^{(4)}$.

A gordura rodeia todas as estruturas orbitais, fornecendo sustentação para o globo e facilitando os seus movimentos. A gordura orbitária é contida pelo septo orbitário. Como uma das alterações involutivas que ocorrem no organismo, o septo orbitário se torna mais fino, permitindo o prolapso da gordura orbitária que pode ocorrer no canto externo, assim como em outras localizações.

Estas duas entidades - dermolipoma e prolapso de gordura orbitária - possuem nosologia que permite a diferenciação clínica de ambas, muito embora a aparência clínica de ambas seja muito semelhante.

A grande maioria dos pacientes é da raça branca $(93,3 \%)$. Os outros relatos $^{(1-2)}$ não apontam diferença entre raças, e este achado pode estar relacionado ao fato do predomínio da raça branca em nossa região.

O dermolipoma foi encontrado mais em mulheres e o prolapso de gordura orbital, em homens. O número de observações não permite que se conclua sobre isso, apesar da literatura também apontar predominância de acometimento de homens quando se trata de prolapso da gordura orbitária ${ }^{(2)}$.

Quanto à idade, o dermolipoma caracteristicamente está presente ao nascimento (o que ocorreu em 10 dos 20 casos) ou é notado nos primeiros anos de vida. Já, o prolapso surge na terceira idade, como também acorreu com nossos pacientes. Existe a possibilidade de indivíduos mais novos apresentarem prolapso da gordura orbitária, desde que haja história de trauma pregresso ${ }^{(2)}$.

Quanto à localização, as duas entidades ocorrem mais no canto externo.

Por ser o dermolipoma um tumor disgenético, é possível que ocorram outras alterações associadas, como foi constatado em alguns de nossos pacientes. Na literatura aparecem casos associados a várias síndromes como Goldenhar e Duane ${ }^{(5-6)}$. 


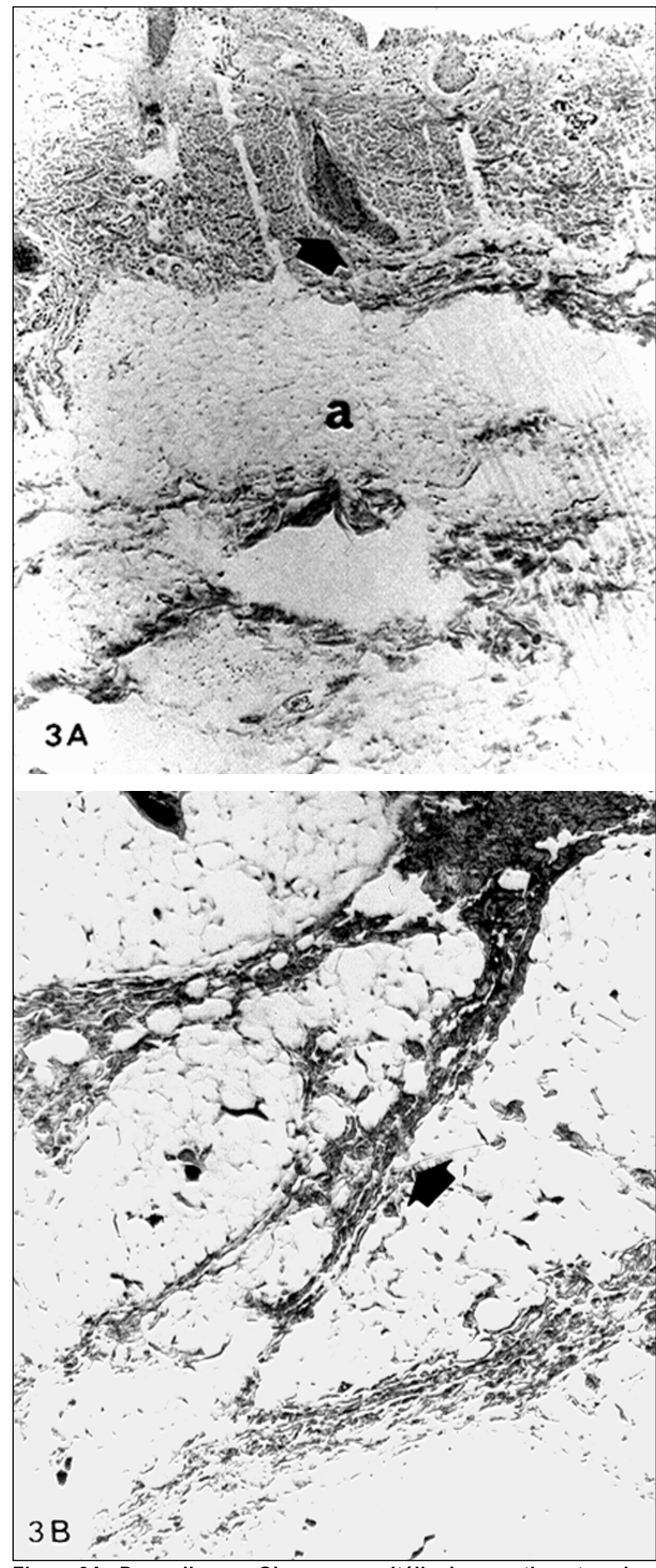

Figura 3A - Dermolipoma. Observar o epitélio de revestimento sobre estroma colágeno denso, contendo estruturas pilosebáceas (seta). Adipócitos (a) no estroma profundo.(X20 - HE); B - Dermolipoma. Os lóbulos de adipócitos estão separados por septos fibrosos (seta). (X 40 - HE)

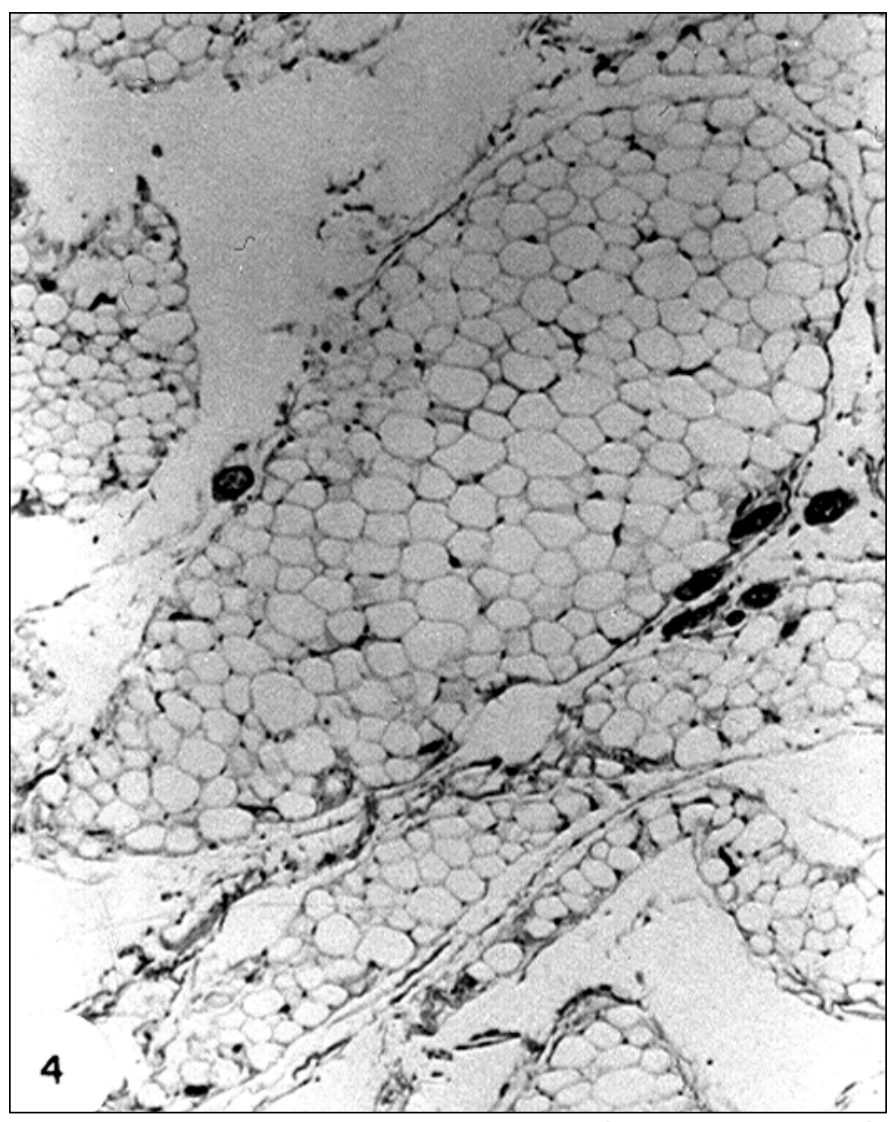

Figura 4 - Prolapso de gordura orbital. A lesão é composta por adipócitos maduros. (X 40 - HE)

A queixa principal que trouxe o paciente ao médico foi a presença da lesão. Alguns pacientes se queixaram de ardor, prurido, desconforto a movimentação ocular lateralmente, e até queixas não associadas como olho seco.

A exérese da lesão, tanto o dermolipoma, como o prolapso de gordura orbitária, não é necessária. Muitas vezes é realizada por questão estética ${ }^{(7)}$, tendo sido efetuada em 14 dos indivíduos aqui estudados.

As lesões muitas vezes se estendem para a órbita. Porém, somente a retirada da porção anterior e aparente das lesões é a conduta mais correta.

Histologicamente o dermolipoma é formado por agregado de adipócitos maduros, envoltos por uma cápsula. Podem ser lobulados e freqüentemente estende-se superior e posteriormente entre o músculo reto lateral e superior, onde está próximo da glândula lacrimal; pode estender-se posteriormente para a órbita ou anteriormente em direção a córnea ${ }^{(8)}$.

A superfície epitelial é freqüentemente fina e lisa, mas ocasionalmente surgem irregularidades; epitélio é escamoso estratificado e parcialmente queratinizado; o estroma contém feixes de colágeno denso, similar ao do dermóide límbico. Estruturas pilosebáceas podem ser raramente observadas. Em algumas lesões o estroma contém cartilagem e glândulas ácinas ${ }^{(9)}$. Já, o prolapso de gordura orbital corresponde histologicamente a apenas adipócitos maduros, dispostos em lóbulos. 
Observou-se dois casos de simbléfaro e pseudopterígio nos pacientes que se submeteram a cirurgia. Muitos relatam complicações com a retirada do dermolipoma, como simbléfaro, ptose, diplopia de difícil tratamento, lesão da glândula lacrimal ou do sistema de drenagem lacrimal e outros sintomas indesejáveis ${ }^{(10-13)}$. Portanto, deve-se atentar para o fato do real benefício que o paciente irá conquistar com a cirurgia.

Além do diferencial entre as duas entidades, objeto deste estudo, podem também ser confundidos com cisto dermói$\mathrm{de}^{(3)}$. Dois pacientes vistos em nosso serviço tiveram diagnóstico clínico sugestivo de dermolipoma e apresentaram ao histopatológico, diagnóstico de cisto dermóide.

Vale lembrar também o diferencial com herniação de glândula lacrimal, condição rara, que ocorre mais em negros e tem associação com blefarocálase ${ }^{(14)}$. Porém, dos nossos pacientes 2 eram negros, um portador de dermolipoma e outro de prolapso de gordura orbital.

Quando a lesão tem localização nasal, o que é incomum e ocorreu em apenas um de nossos pacientes, o diferencial se faz com os tumores desta região: pterígio, pseudopterígio, papiloma e carcinoma espinocelular ${ }^{(15)}$.

\section{CONCLUSÃO}

Os aspectos apontados mostram que o dermolipoma e o prolapso de gordura orbital, apesar de possuírem aparência semelhante, são entidades distintas, sendo possível diferenciá-las clinicamente.

\section{ABSTRACT}

Purpose: To describe the clinical features of patients with dermolipoma and orbital fat prolapse. Methods: A retrospective 12-years survey was done concerning patients with dermolipoma and orbital fat prolapse, at the Faculdade de Medicina de Botucatu - UNESP. Results: During that time we found 20 patients with dermolipoma and 10 with orbital fat prolapse. Dermolipoma occurred mainly in children, more in female, and was frequently located at the external canthus, 7 patients had other ocular associated diseases. The orbital fat prolapse occurred also in Caucasians, more in males, the elderly, and in all but one occurred temporally. Conclusion: Dermolipoma and orbital fat prolapse have similar clinical appearances, but they are clearly distinct. Dermolipoma affects children, female, and orbital fat prolapse, elderly and males.

Keywords: Lipoma; Adipose tissue; Choristoma/diagnosis; Prolapse; Orbital disease/diagnosis; Differential diagnosis; Retrospective studies.

\section{REFERÊNCIAS}

1. Kim YD, Goldberg RA. Orbital fat prolapse and dermolipoma: two distinct entities. Korean J Ophthalmol 1994;8:42-3.

2. McNab AA. Subconjunctival fat prolapse. Aust N Z J Ophthalmol 1999;27:33-6.

3. Eijpe AA, Koornneef L, Bras J, Verbeeten BJ, Peeter FL, Zonneveld FW. Dermolipoma: characteristic CT appearance. Doc Ophthalmol 1990;74:321-8.

4. Jakobiec FA, Jones IS. Mesenchymal and fibro-osseous tumors. In: Tasman W, Jaeger EA editors. Duane's Ophthalmology. New York: Lippincontt-Raven; 1997. vol. 2. cap. 44.

5. Verma MJ, Faridi MM. Ocular motility disturbances (Duane retraction Syndrome and doublé elevator palsy, with congenital heart disease, a rare association with Goldenhar syndrome - a case report). Indian J Ophthalmol 1992;40:61-2.

6. Craitoiu S. [Goldenhar's oculoauricular dysplasia, limbic dermoid and conjuntival dermolipoma]. Oftalmologia 1992;36:357-61.

7. Alves MR, Kara-José N. Conjuntiva cirúrgica. São Paulo: Roca; 1999. 255p.

8. Cotran RS. Soft tissue tumors and tumor-like lesions. In: Cotran RS, Kumar V, Robbins SL. Robbins pathologic basis of disease. $5^{\text {th }}$ ed. Philadelphia: Saunders; 1994.

9. Spencer WH. Congenital and developmental abnormalities. In: Spencer WH Ophthalmic pathology: an atlas and textbook. 3.ed. Philadelphia, W.B. Saunders; 1987. p.121-9.

10. Economidis I, Tragakis M, Mangouritsas N, Papademetriou D. Keratoconjuntivitis sicca following excision of a dermolipoma of the lacrimal gland. Ann Ophthalmol 1978;10:1273-8.

11. Fry CL, Leone CR Jr. Safe management of dermolipomas. Arch Ophthalmol 1994;112:1114-6.

12. François P, Lekieffre M, Woillez M, Ryckewaert M. Complications of dermolipoma ablation. Bull Soc Ophthalmol Fr 1989;89:289-90.

13. Beard C. Dermolipoma surgery or "an ounce of prevention is worth a pound of cure". Ophthal Plast Reconstr Surg 1990;6:153-7.

14. Smith B, Petrelli R. Herniation of the lacrimal glands. Trans Am Acad Ophthalmol Otolaryngol 1997;84:988-90.

15. Waller SG, Adamis AP. Pterygium. In: Tasman W, Jaeger EA editors. Duane's Ophthalmology. New York: Lippincont-Raven; 1997. vol. 6 cap.35.

\title{
ABO ELETRÔNICO
}

\author{
Novo site
}

\section{Acesso: http://www.abonet.com.br}

